\title{
The effect of a hot-wet environment on adhesively bonded joints under a
}

\author{
sustained load \\ X Han ${ }^{1,2}$, AD Crocombe ${ }^{1, *}$, SNR Anwar ${ }^{1,3}, \mathrm{P} \mathrm{Hu}^{2}, \mathrm{WD} \mathrm{Li}^{2}$ \\ ${ }^{1}$ Mechanical Engineering Science, University of Surrey, Guildford, UK \\ ${ }^{2}$ School of Automotive Engineering, Dalian University of Technology, Dalian, PR China \\ ${ }^{3}$ Civil Engineering, University of Mataram, Mataram, Indonesia \\ * Corresponding author. Tel.: +44 01483689194 \\ E-mail address: a.crocombe@surrey.ac.uk (AD Crocombe).
}

Keywords: Epoxy; Finite element analysis; Adhesion by diffusion; Aging; Aluminium and alloys

\begin{abstract}
The aim of this research was to develop numerical modelling techniques for simulating the simultaneous effects of moisture, elevated temperature and applied load on the performance of adhesively bonded joints. Associated experimental data are also reported. The degradation process of the joints was modelled using a fully-coupled approach, with the moisture concentration affecting the stress distribution and the stress state affecting the moisture diffusion analyses simultaneously. Further, the stress analysis contains a moisture dependent creep model to accommodate viscous effects and both swelling and thermal strains were included in the simulation. The governing parameters adopted in the modelling procedure were determined from experimental work based on the bulk adhesive. The joint response was monitored throughout the ageing process and good correlation was found between the experimental and numerical results.
\end{abstract}

\section{Introduction}

Adhesives have been increasingly used in modern structural engineering with specific benefits including more joining flexibility and a better stress distribution and fatigue resistance [1-3]. One of the major concerns which inhibit a more wide-spread application of adhesive bonding is its prolonged durability in hostile service conditions, including moisture absorption, residual shrinkage, sustained mechanical, thermal and swelling stresses and the concomitant joint degradation [3-6].

In earlier studies, Kinloch [7] stated that the mechanical performance of adhesive joints may be adversely affected when exposed to aqueous environments, especially at an elevated temperature. Research by Adams et al. [1] revealed that thermal stresses coupled with the external loads influenced the mechanical behaviour of adhesive joints. Brewis et al. [8] studied single lap joints bonded with an epoxide-polyamide adhesive exposed to a warm moist environment for up to 2500 hours and concluded that the moisture degradation was due to plasticisation of the adhesive.

Crocombe [9] developed a framework (interfacial and cohesive) to assess the effect of 
environmental degradation and subsequent cohesive failure in adhesively bonded structures. Finite element simulation was carried out on joints bonded with FM1000 (Cytec ${ }^{\circledR}$, New Jersey, USA) exposed to water over periods of time assuming Fickian moisture diffusion. Many subsequent researchers focused on the combined experimental-numerical method to investigate the performance of adhesive joints under a hot-humid environment and developed a range of prediction methodologies [10-13]. Liljedahl et al. [14] studied a sequentially-coupled stress-diffusion analysis in bonded steel joints. It was found that the residual stresses relaxed significantly over time and did not further degrade the joint strength significantly. Sugiman et al. [15-16] presented a combined experimental-numerical approach to investigate the static and fatigue behaviour of adhesively bonded structures immersed in deionised water at $50{ }^{\circ} \mathrm{C}$ for up to 2 years. Experiments revealed that joint strength and fatigue life decreased with increasing moisture content and levelled off towards saturation. $\mathrm{Hu}$ et al. [17] investigated the effects of cyclic thermal loading with a cycle period of 2 hours on adhesively bonded joints for up to 8 weeks. A degraded cohesive zone model was selected to simulate the degradation process in numerical modelling and good agreement was achieved with the experimental data. Banea et al. [18] discussed the effect of temperature on Mode I fracture toughness of adhesive joints through both experimental and numerical methods. Results showed that adhesives have mechanical properties that vary with temperature and the simulated predictions of the joint fracture toughness matched the experiment results well. Abdel Wahab et al. [19] developed an experimental-analytical-numerical method to study the moisture diffusion in adhesively bonded composite structures under different degradation conditions (temperature, relative humidity). With the diffusion coefficients obtained from experiment, good agreement was found between the analytical and numerical results.

However, to date, coupled situations where stress and moisture uptake occur simultaneously have not been studied. In this current work, prior to numerical simulation, experimental work was undertaken on bulk adhesive and adhesive joints to measure a) the stress dependency of moisture diffusion, b) the moisture dependent adhesive stress-strain and creep compliance curves. Based on the measured adhesive material data, numerical modelling was then carried out to simulate the long-term degradation process in adhesively bonded joints under simultaneously combined thermal-hygro-mechanical loading conditions using a fully-coupled methodology.

\section{Experimental procedures and results}

\subsection{Specimen fabrication}

FM73 (Cytec ${ }^{\circledR}$, New Jersey, USA) film adhesive with a knit carrier and nominal thickness of $0.18 \mathrm{~mm}$ was utilised to make bulk adhesive dogbone and single lap joint (SLJ) specimens. The required bulk adhesive specimens were obtained by stacking 9 layers of FM73 adhesive film. A specimen thickness of $1 \mathrm{~mm}$ was maintained using steel spacers. The curing process was performed at the recommended curing temperature of $120{ }^{\circ} \mathrm{C}$ [20]. After curing, dumbbell specimens of overall length of $65 \mathrm{~mm}$ and gauge length and width of $30 \mathrm{~mm}$ and $5 \mathrm{~mm}$ respectively were cut with a computer numerical control (CNC) machine. Details related to the curing process can be found elsewhere [15, 21-22]. 
Aluminium alloy 2024-T3 was used as the substrates in the single lap joints, shown in Fig 1. Prior to curing, the aluminium alloy substrates were surface treated to provide a durable interface. The treatment technique started with chromic acid etching (CAE) followed by phosphoric acid anodising (PAA) and then the application of corrosion inhibiting BR127 (Cytec $^{\circledR}$, New Jersey, USA) primer [23-24]. Two layers of adhesive film, having the same dimensions as the overlap area, were laid up on the pre-treated aluminium surfaces. The adhesive joints were then stacked one on top of the other and cured in a spring-loaded jig. A spacer $0.2 \mathrm{~mm}$ thicker than the substrate was inserted between the specimens to maintain a bond line thickness of $0.2 \mathrm{~mm}$. The curing procedures were the same as for the bulk adhesive described above. The detailed configuration of the single lap joint is shown in Fig. 1.

\subsection{Experimental method}

\subsubsection{Bulk adhesive}

The bulk adhesive dumbbell specimens were immersed in water at $50^{\circ} \mathrm{C}$ (both unloaded and at $25 \%$ of the static failure load) for 6 months to obtain various material properties, including (a) moisture ingress under different loading levels, (b) the creep response, and (c) hygroscopic expansion (swelling) coefficients (CHE). The loads were applied using the spring loaded rig shown in Fig 2. This rig was placed in a tank containing the water, which in turn was placed in an oven at $50^{\circ} \mathrm{C}$.

A gravimetric method was used to obtain the adhesive moisture diffusion coefficients and equilibrium moisture uptake, assuming the Fickian diffusion model as expressed in Eq. 1 [25].

$$
F_{x}=-D \frac{d c}{d x}
$$

Here, $F_{x}$ is the flux of moisture in $x$ direction, $D$ is the diffusion coefficient and $d c / d x$ is the normalised moisture concentration gradient in the $x$ direction. Details of this procedure can be found elsewhere [26]. Loaded specimens were periodically removed from the spring loaded rig for gravimetric measurements.

The creep extension of the adhesive was obtained by measuring the extension of the pre-compressed spring. This was generally undertaken immediately before removing the specimen for gravimetric testing. A power-law creep model was used in the subsequent modelling procedure as expressed in Eq. 2 [27].

$$
\dot{\varepsilon}=A q^{n} t^{m}
$$

Here, $\dot{\varepsilon}$ represents the creep strain rate, $q$ is the equivalent stress, $t$ is the time and $A, n$ and $m$ are coefficients based on a fitting procedure to the experimental data.

Additional quasi-static tensile tests to failure have also been carried out on bulk adhesive specimens that had been pre-saturated to different levels [28]. Moisture dependent stress-strain curves were obtained from the resulting data.

The CTE of FM73 adhesive was determined using strain gauges on both the adhesive and

a reference material (aluminium 2024-T3 with a CTE of 2.36E-5 ${ }^{\circ} \mathrm{C}^{-1}$ ) [28]. Both materials with strain gauges attached were placed in the oven to measure the strain variation with 
increased temperature in both specimens at the same time. The relationship between the CTEs for the adhesive and the reference material can be deduced from Eq. 3 [29].

$$
\alpha_{A}-\alpha_{R}=\frac{\left(\varepsilon_{T / O /(G / S)}-\varepsilon_{T / O /(G / R)}\right)}{\Delta T}
$$

Here, $\alpha_{A}$ and $\alpha_{R}$ are CTEs for the adhesive and reference materials, $\varepsilon_{T / O /(G / S)}$ and $\varepsilon_{T / O /(G / R)}$ are strain outputs for adhesive and reference and $\Delta T$ is the temperature change from the initial reference temperature. The hygroscopic expansion in the adhesive was assumed to be isotropic and a micrometer was used to measure the increase in the thickness of the unloaded bulk adhesive dumbbell specimens during the degradation process [22, 30].

\subsubsection{Single lap joint}

Creep tests on adhesively bonded joints were carried out in similar spring-loaded jigs. However, with these tests each specimen was encased in a glass tube filled with de-ionised water and the resulting assembly placed in an oven at $50^{\circ} \mathrm{C}$. Different loading levels $(12.0 \%$ and $17.5 \%$ of the dry joint failure strength) were used to investigate the effect of stress on the joint response. The joint extension was determined by periodic measurement of the displacement of the pre-compressed spring.

\subsection{Experimental results}

Experimental results based on bulk adhesive tests provided CTE, CHE, moisture dependent stress-strain data, stress dependent moisture diffusion and moisture dependent creep behaviour for the subsequent numerical modelling. The CTE and CHE for FM73 adhesive were found to be $8.00 \mathrm{E}-5^{\circ} \mathrm{C}^{-1}$ and $0.00463\left(\% m_{w}\right)^{-1}\left(\% m_{w}\right.$ being the percentage mass of water in the adhesive by weight) respectively. The effect of moisture on the adhesive Young's modulus is given in Table 1. It can be seen that the Young's modulus reduces by about $15 \%$ due to the presence of moisture. Young's modulus and CTE used for the 2024-T3 aluminium alloy are $70 \mathrm{GPa}$ and $2.36 \mathrm{E}-5^{\circ} \mathrm{C}^{-1}$ respectively.

The dependency of the Fickian moisture diffusion parameters on the stress state are shown in Table 2. The moisture diffusion coefficients are significantly affected by the applied stress leading to a more rapid moisture uptake. Further, sustained loading is seen to increase the saturated mass uptake of water by over $25 \%$ from the unloaded condition. The increase in both parameters might be explained by the increase in the free volume of the bulk adhesive under load and/or the presence of crazes [31-32]. Both aspects may be caused by the sustained loading these specimens experience.

The creep properties of the adhesive in the ageing environment under $25 \%$ of the static failure load are shown in Table 3. These were determined based on the results from dry and pre-saturated bulk adhesive creep tests [27] and data from the gradually saturated bulk adhesive dumbbell specimen discussed above. The gradually saturated bulk adhesive experimental and numerical creep-time curves are consistent with each other as presented in Fig. 3. A two-phase (primary and secondary) creep model was used to provide better fitting with the experimental data. 
Creep behaviours based on adhesively bonded joints under two loading levels were obtained and used as a validation of the numerical simulation. The experimental creep strain-time curves are shown later in Fig. 10d, where they are compared with the predicted response

\section{Finite element modelling}

A 3D finite element (FE) model was built in the FE package Abaqus ${ }^{\circledR}$ to simulate the fully-coupled degradation process of the adhesive joint carrying a sustained load in a hot-humid environment. Material parameters considered in this numerical modelling were obtained through the experiments discussed in Section 2 and this section focuses on incorporating the relevant behaviours into the FE model.

The numerical analysis was carried out assuming geometric non-linearity. Fig. 4 shows a typical FEM mesh for the SLJ, with detailed refinement around the bonding areas, where large peel and shear stress gradients are located [33]. Standard quadratic 3D stress elements (C3D20) were employed for the substrates around the bonding area to provide a more accurate stress description whilst reduced integration elements (C3D20R) were used for the rest of the substrate to reduce the computational cost. Quadratic 3D thermal-displacement coupled elements (C3D20T) were used for the adhesive to include both moisture diffusion (using a heat transfer analogue) and stress. To obtain more accurate results, a higher mesh density $(0.20 \mathrm{~mm} \times 0.20 \mathrm{~mm} \times 0.17 \mathrm{~mm})$ was adopted for the elements around the bonding area.

One end of the substrate was assigned an encastre constraint, while a kinematic coupling was applied to impose the same axial displacement on all the nodes at the other end of the joint, with the displacements of these nodes in the other two directions constrained. A concentrated load was applied at the control point of the kinematic coupling to simulate the load supplied by the springs in the experiment procedure. Moreover to simulate an immersed environment, the exposed surfaces of the adhesive layer were assigned an initial normalised moisture concentration (temperature in Abaqus) boundary condition of 1 at all exposed adhesive faces, as shown in Fig. 4.

\subsection{Stress dependent moisture diffusion}

An analogy between heat transfer and moisture diffusion was utilised in this model to simulate the moisture ingress process [34]. Further details of the diffusion analysis can be found elsewhere [26]. In order to simulate the simultaneous coupled effect of moisture and stress on each other, fully coupled thermal-displacement elements were utilised for the adhesive layer. Using an Abaqus user subroutine USDFLD, the von Mises stress for each adhesive material (integration) point was defined as a field variable (FV1) and the diffusion coefficient was defined as being linearly dependent on FV1 (the stress), as given in Table 2.

\subsection{Moisture dependent elasticity}

As found in the bulk adhesive tensile tests, the adhesive modulus is a function of moisture. In this model, the moisture dependent adhesive modulus data shown in Table 1 was 
incorporated by defining the moisture content as another field variable (FV2) in the user subroutine USDFLD. This is determined by the normalised moisture concentration, the stress dependent saturation uptake (Table 2) and the von Mises stress. The Young's modulus of the adhesive was defined as being linearly dependent on this field variable.

\subsection{Thermal and swelling expansions}

The thermal expansion of both the adhesive and the substrate were introduced through the user subroutine UEXPAN which produces a total "expansion" strain. A temperature drop of $-50{ }^{\circ} \mathrm{C}$ was defined as a predefined field variable (FV3) and used with the CTE to define the thermal strains. This temperature drop represents the decrease from the adhesive glass transition temperature $\left(100{ }^{\circ} \mathrm{C}\right)$ to the environmental testing temperature $\left(50{ }^{\circ} \mathrm{C}\right)$. Meanwhile, the swelling strain of the adhesive was defined as the product of the moisture content (FV2) and the CHE. Values of the expansion coefficients are given at the beginning of Section 2.3.

\subsection{Moisture dependent time-hardening creep}

As described in Section 2, a two-phase time-hardening power law creep model was used and the parameters shown in Table 3 were adopted to define the adhesive creep behaviour. These parameters are dependent on both moisture and the creep strain (transition at $48 \%$ from primary to secondary creep). With the moisture content defined as a field variable (FV2) and the creep strain as another field variable (FV4) in user subroutine USDFLD, the two-phase moisture dependent creep model parameters were defined as being dependent on these field variables. The dependence was linear with moisture and stepwise at the transition strain (primary to secondary creep).

\subsection{Concept framework of the FE modelling}

A general framework is shown in Fig. 5, illustrating the modelling techniques outlined above. This should clarify what is a quite a complex modelling concept. It can be seen that the four field variables described above are determined in increment $\mathrm{N}-1$ and imported into the next increment. Four adhesive behaviours (moisture diffusion, elasticity, creep and thermal and swelling expansion) in increment $\mathrm{N}$ are considered and determined using the field variable values from the previous step. Then the FE calculation is carried out for the SLJ model, providing an updated stress and moisture distribution which is then used to calculate the field variable values in the subsequent increment. This iterative process is repeated until the target simulation time (i.e. 3 or 6 months) is achieved.

\section{FE modelling results and discussion}

Two steps were utilised in this Abaqus model to provide results data at the specific time points of 3 and 6 months during the environmental ageing process. The separate field variable dependent modelling aspects outlined in Sections 3.1 to 3.4 were all validated successfully using simple benchmarking tests. In this paper the focus is on the whole joint modelling, which incorporates all these features rather than the validating benchmarking tests. Three simulations (unloaded, creep at $12.0 \%$ and at $17.5 \%$ of the static failure strength respectively) were performed to investigate the effects of stress relaxation caused by moisture dependent creep and thermal and swelling expansion. 


\subsection{Moisture ingress}

Normalised moisture concentration, varying from 0 to 1 , is a dimensionless description of the moisture profile in the adhesive; with the completely dry condition corresponding to 0 and the saturated condition to 1 . The normalised moisture concentration contours on the adhesive mid-plane for the joint loaded at $12 \%$ of the static failure strength at initial, 12 and 42 days ageing are presented in Figs. 6a-6c respectively. It is observed that no moisture exists in the centre of the adhesive initially while after 12 and 42 days water diffuses into the adhesive layer and the normalised moisture concentrations in the centre reach around 0.4 and 0.8 respectively. The distribution of the normalised moisture concentration along a path travelling through the centre of the adhesive in a widthwise direction is shown in Fig. 6d. It can be observed that the adhesive layer in the single lap joint reaches full saturation after 3 months exposure.

The distributions of the actual (not normalised) moisture content (FV2) in both unloaded and loaded (12\% of failure strength) conditions along the same path as Fig 6d are shown in Fig. 7. It is observed that the stress dependent moisture diffusion process is slowed in the absence of an external load. However, the unloaded moisture content level is not reduced by the amount suggested from the saturation mass uptakes in Table 2. This is due to the internal stress caused by the thermal and swelling expansion further clarified in Section 4.2.

\subsection{Stress distribution}

The von Mises stress has been used to evaluate the variation of adhesive stress in the SLJ with time. The von Mises stress contours on the mid-plane of the adhesive at initial state, 12 days and 42 days are shown in Figs $8 a-8 c$ for the joint loaded at $12 \%$ of the static failure strength. Fig 8d shows the same stresses along a path in the middle of the adhesive layer running in the lengthwise direction initially, after 12 days and 42 days. It can be seen from Fig 8d that the stress concentration usually observed at the end of the bonding area is initially reversed (12 days) and then largely reduced after 3 months environmental degradation. The initial reversal can be explained by the rapid development of creep strains in the highly stressed region at the overlap ends. This increase in creep stains will reduce the elastic strains and hence the stresses. The subsequent smoothing of the von Mises stresses can be explained by a combination of the same mechanism and the fact that as the moisture diffuses further into the joint there is less resistance to the swelling by the dry inner core. As can be seen in Table 3 , the creep parameters are dependent on the moisture content level and the stress and this leads to a much higher creep rate on the edges of the adhesive layer. Therefore, more stress relaxation occurs in the outer region thus averaging the stress distribution in the bonding area. After 3 months the adhesive layer is essentially saturated and the stress is nearly uniform, thus the joint continues to creep at a broadly uniform rate across the entire adhesive layer.

Similar investigation was also carried out on the joint model without any applied load and the von Mises stress contours on the mid-plane of the adhesive are shown in Figs 9a-9c. Also included are stresses along a path in the middle of the adhesive layer running in the lengthwise direction initially, after 12 days and 42 days as shown in Fig. 9d. It can be seen 
from Figs. 9a-9c that the overall stress level is not significantly reduced when no mechanical load is applied. This is both because the mechanical load is only $12 \%$ of the static failure load and because some components of the mechanical and swelling/thermal stresses act in opposite directions. The residual stress in the adhesive layer is caused by the thermal and swelling expansion. The temperature difference imported initially into this model leads to non-negligible internal stress when the CTEs in the adhesive and substrate are dissimilar, whilst the swelling strains, which are proportional to the moisture content, exist throughout the ageing process. It can be seen from Fig. 8d and Fig. 9d that a similar stress relaxation process is observed. However, the difference lies in that in the unloaded model the stress level keeps decreasing with accumulated creep and swelling expansion, whilst in the loaded model a certain lower limit can be achieved due to the applied load.

\subsection{Creep behaviour}

The equivalent creep strain is used in this section to evaluate the joint deformation with time. The simulation of the creep behaviour, which is dependent on the moisture content in the adhesive as well as the stress, is a key factor in this modelling concept. The equivalent creep strain contours on the adhesive mid-plane at initial state, 3 months and 42 months are illustrated in Figs. 10a-10c for the joint loaded at $12 \%$ of the static failure strength. It is found that the creep strain increases much faster in the first 3 months than the latter 3 months which is reasonable based on the moisture diffusion and creep models which develop more rapidly initially. Further, as the creep model is moisture dependent and the edges of the joint are saturated, maximum creep strains are located at the edges of the adhesive throughout the entire time. With increased creep strain at the overlap ends the stresses in these regions are reduced, relative to the centre of the joint leading to a more uniform stress distribution. This is consistent with the discussion in Section 4.2.

To compare the creep response obtained from the FEM method and the experimental work, the experimentally measured and numerically simulated adhesive joint displacements under the two loading levels are shown in Fig. 8d. Reasonable agreement is observed over the loading period, particularly at the higher creep load.

\section{Conclusions}

A fully-coupled moisture-displacement analysis was carried out in Abaqus to simulate the ageing process in adhesively bonded joints under simultaneously combined thermal-hygro-mechanical loading conditions. The complex coupling that occur between the different adhesive "material" behaviours have been successfully connected by defining field variables in ABAQUS and making the "material" responses a function of these field variables. Experimental work on simultaneously loaded and aged bulk adhesive specimens have provided the material input in this study, while the tests carried out on simultaneously loaded and aged single lap joints provided positive validation with the numerical results.

This work is an important but subsidiary phase to the driving goal of this research which is to investigate the relationship between the accumulated degradation factors (e.g. creep strain and moisture) and the reduction in static strength in adhesive joint after long-term 
exposure to hostile environment providing these degradation factors. This will be achieved by testing the joints that have been aged in this paper and implementing progressive damage FE analysis of quasi-statically loaded joints following the ageing. Again, predicted and measured responses will be compared to assess the validity of the technique.

\section{Acknowledgements}

The authors would like to acknowledge the Key Project of the National Natural Science Foundation of China (No. 10932003) and the China Scholarship Council for the financial support. Thanks are also due to Mr S. McAllister (Bombardier Aerospace) for provision of the adhesive and substrate surface treatments.

\section{References}

[1] Adams R. D., Comyn J., and Wake W. C., Structural adhesive joints in engineering. 2nd ed. (Chapman \& Hall, London, 1997).

[2] Comyn J., Developments in adhesives (Ed. Kinloch AJ.) (Applied Science Publishers, London, 1981).

[3] da Silva L. F. M., Öchsner A., and Adams R. D., Handbook of Adhesion Technology (Heidelberg, Springer, 2011).

[4] Zewi I. G., Flashner F., Dodiuk H., and Drori L., Int. J. Adhes. Adhes. 4, 137-139 (1984).

[5] Wagner H. D., Lustiger A., Compos. 25, 613-616 (1994).

[6] da Silva L. F. M. and Sato C., Design of adhesive joints under humid conditions (Springer, Heidelberg, 2013).

[7] Kinloch A. J., Adhesion and adhesives: science and technology (Chapman \& Hall, London,1983).

[8] Brewis D. M., Comyn J., and Shalash R. J. A., Int. J. Adhes. Adhes. 2, 215-222 (1982).

[9] Crocombe A. D., Int. J. Adhes. Adhes. 17, 229-238 (1997).

[10] Wylde J. W., Spelt J. K., Int. J. Adhes. Adhes. 18, 237-246 (1997).

[11] Hu P., Han X., Li W. D. , Li L., and Shao Q., Int. J. Adhes. Adhes. 41, 119-126 (2013).

[12] Hua Y. Crocombe A. D., Wahab M. A., and Ashcroft I. A., Int. J. Adhes. Adhes. 28, 302-313 (2008).

[13] Hua Y. Crocombe A. D., Wahab M. A., and Ashcroft I. A., J. Adhes. 82, 135-160 (2006).

[14] Liljedahl C. D. M., Crocombe A. D., Wahab M. A., and Ashcroft I.A., J. Adhes. Sci. Technol. 19, 525-547 (2005).

[15] Sugiman S., Crocombe A. D., and Ashcroft I. A., Int. J. Adhes. Adhes. 40, 224-237 (2013).

[16] Sugiman S., Crocombe A. D., and Ashcroft I. A., Int. J. Adhes. Adhes. 41, 80-91 (2013).

[17] Hu P., Han X., da Silva L. F. M. , and Li W. D., Int. J. Adhes. Adhes. 41, 6-15 (2013).

[18] Banea M. D., da Silva L. F. M., and Campilho R. D. S. G., Int. J. Adhes. Adhes. 31, 273-279 (2011).

[19] Abdel Wahab M. M., Ashcroft I. A., Crocombe A. D., and Shaw S. J., J. Adhes. 77, 43-80 (2001).

[20] Cytec Engineered Materials. FM® 73 Toughened Epoxy Film. Technical Service. 1300 Revolution Sheet, Harve de Grace, Maryland; 1998.

[21] Sugiman S., Crocombe A. D., and Ashcroft I. A., Engng. Fract. Mech. 98, 296-314 
(2013).

[22] Liljedahl C. D. M., Crocombe A. D., Wahab M. A., and Ashcroft I. A., J. Adhes. 82, 1061-1089 (2006).

[23] ASTM D2674-72. Standard methods of analysis of sulfochromate etch solution used in surface preparation of aluminum. West Conshohocken (PA): ASTM International, 2012.

[24] ASTM D3933-98. Standard guide for preparation of aluminum surfaces for structural adhesives bonding (phosphoric acid anodizing). West Conshohocken (PA): ASTM International, 2010.

[25] Crank J., The mathematic of diffusion (Oxford University Press, London, 1975).

[26] Sugiman S., Combined environmental and fatigue degradation of adhesively bonded metal structures. PhD thesis. University of Surrey, Guildford, UK;2011.

[27] Liljedahl C. D. M., Crocombe A. D., Wahab M. A., and Ashcroft I. A., Int. J. Adhes. Adhes. 27, 505-518 (2007).

[28] Liljedahl C. D. M., Modelling the interfacial degradation in adhesively bonded joint. PhD Thesis. University of Surrey, Guildford, Surrey, UK;2006.

[29] Measurement group, Inc. Tech note TN-513-1. Measurement of thermal expansion coefficient using strain gages. Raleigh, North Carolina, USA; 1986.

[30] Liljedahl C. D. M., Crocombe A. D., Wahab M. A., and Ashcroft I. A., Int. J. Fract. 141, 147-161 (2006).

[31] Neumann S. and Marom G., J. Mat. Sci. 21, 26-30 (1986).

[32] Comyn J., Durability of Structural Adhesives (Ed. Kinloch AJ.) (Applied Science Publishers, London, 1983).

[33] Panigrahi S. K. and Pradhan B., J. Reinforc. Plast. Compos. 26, 183-201 (2007).

[34] Szekeres A., Comput. Struct. 76, 145-152 (2000).

\section{Figures}

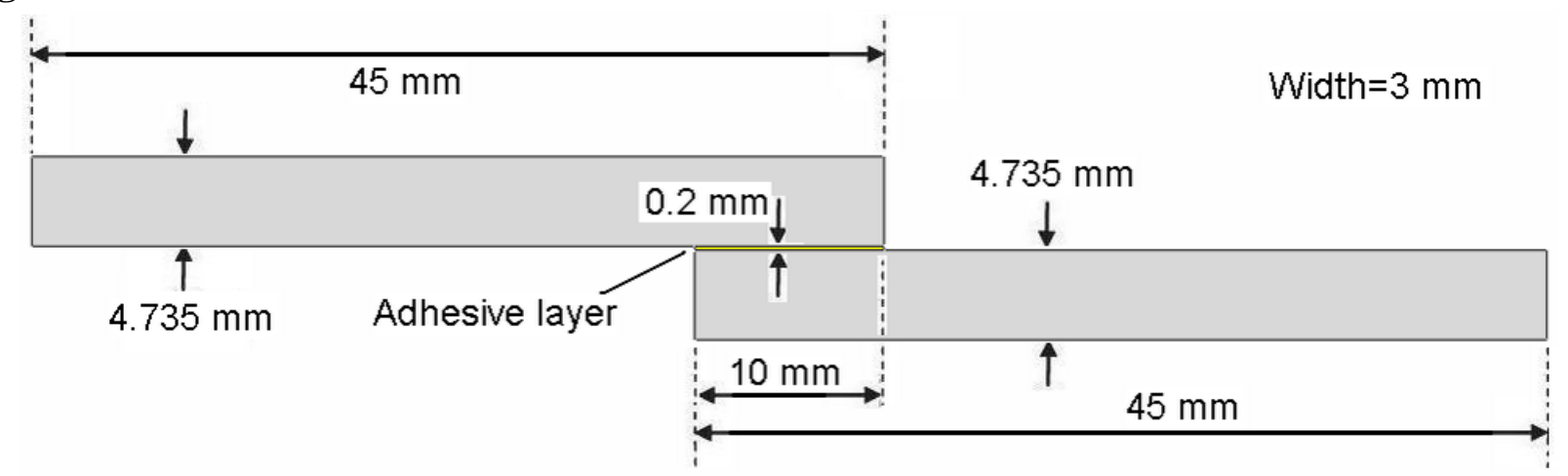

Fig. 1. Configuration of the single lap joint. 


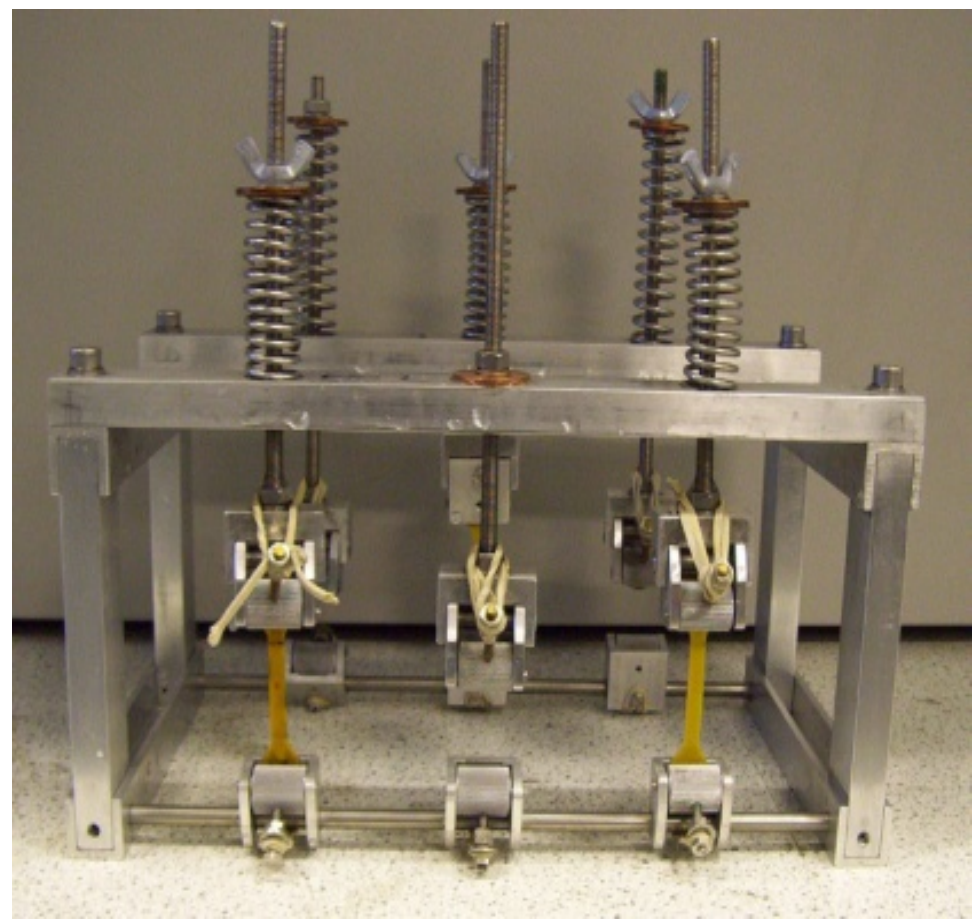

Fig. 2. Loading jigs for the bulk adhesive degradation test.

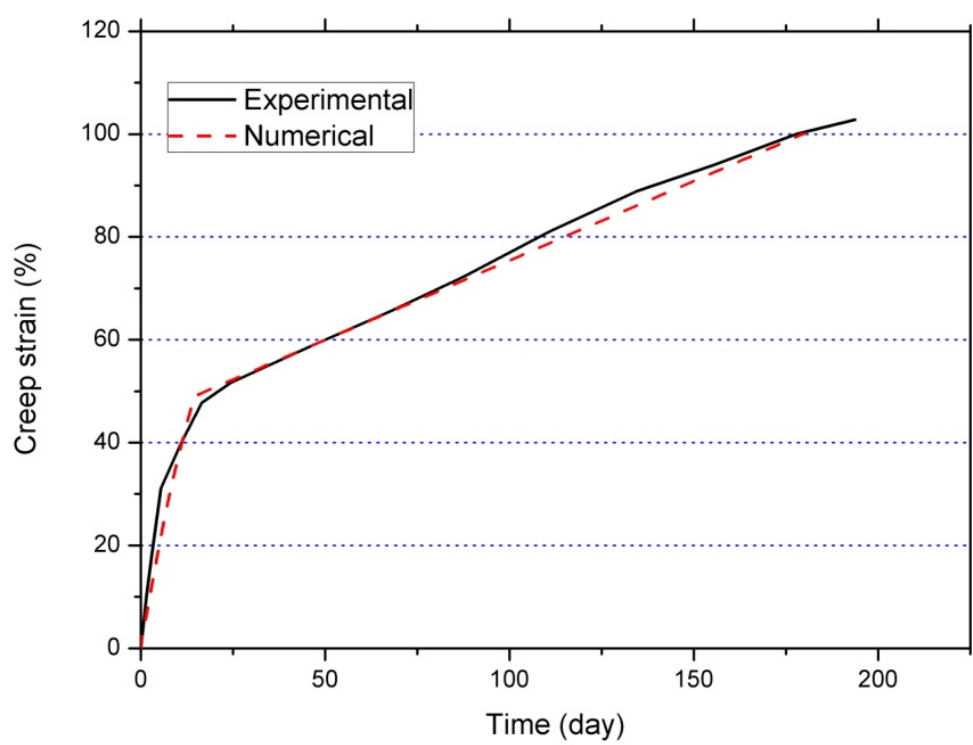

Fig. 3. The numerical and experimental creep strain-time curves at $50^{\circ} \mathrm{C}$ and under $25 \%$ of the static failure load for FM73 bulk adhesive. 


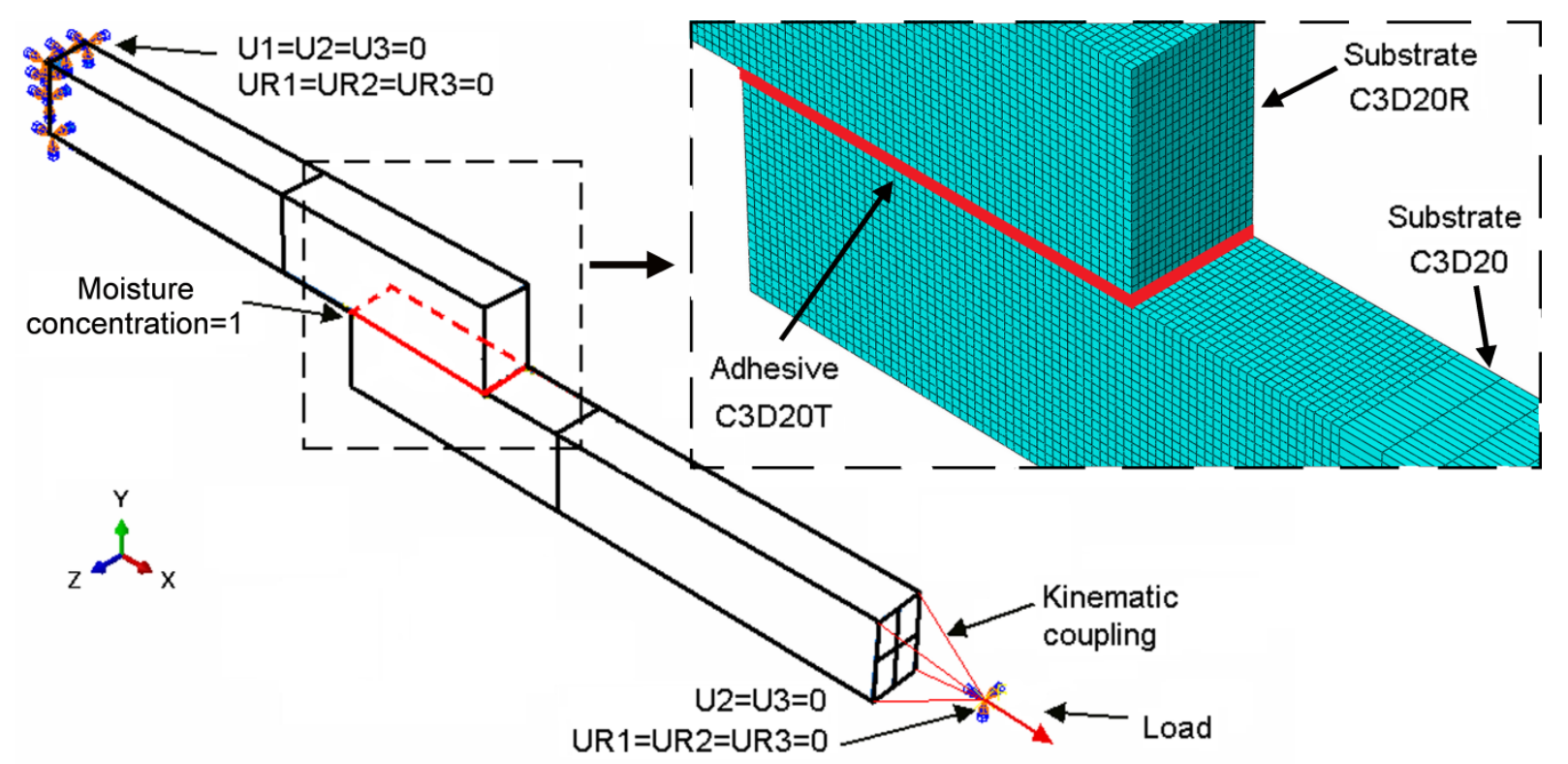

Fig. 4. FE mesh for the single lap joint showing detailed refinement around the bonding area and boundary conditions.

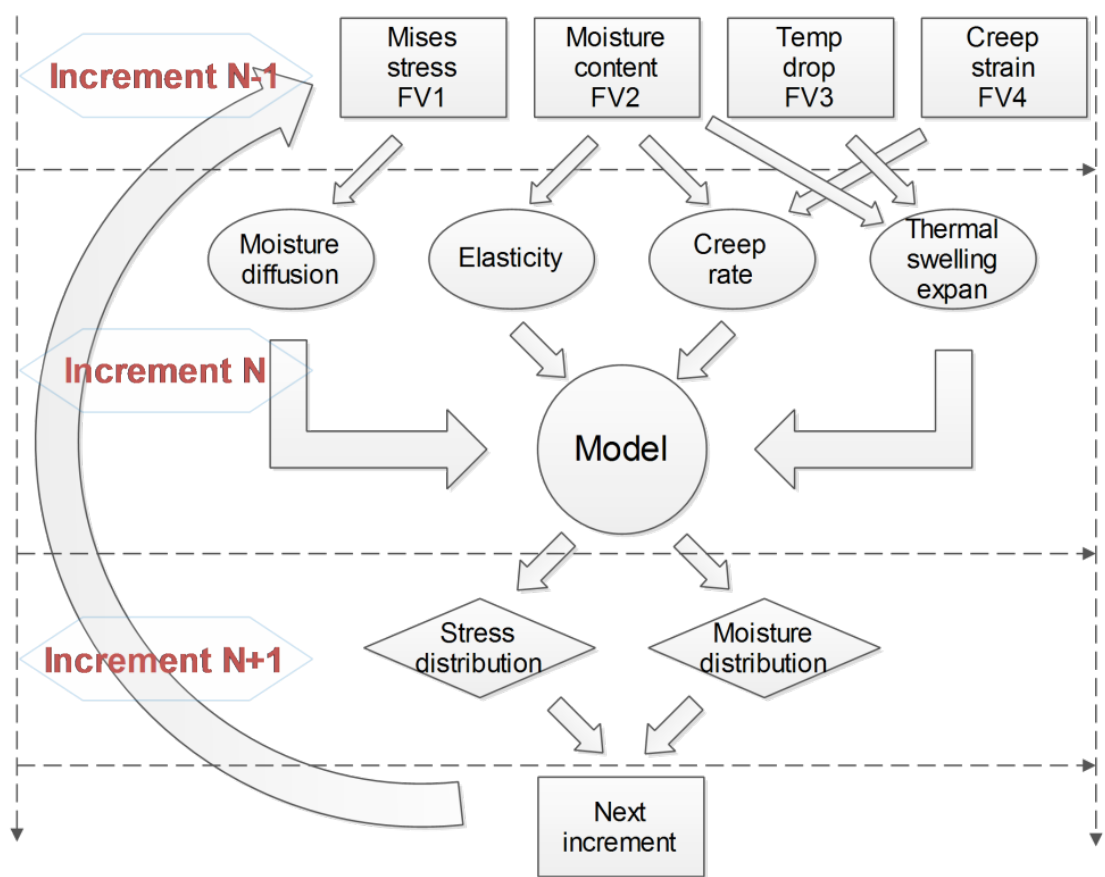

Fig. 5. Concept framework of the FE modelling. 


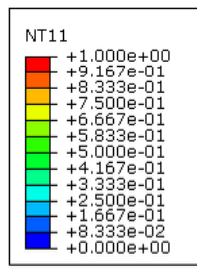

(a)

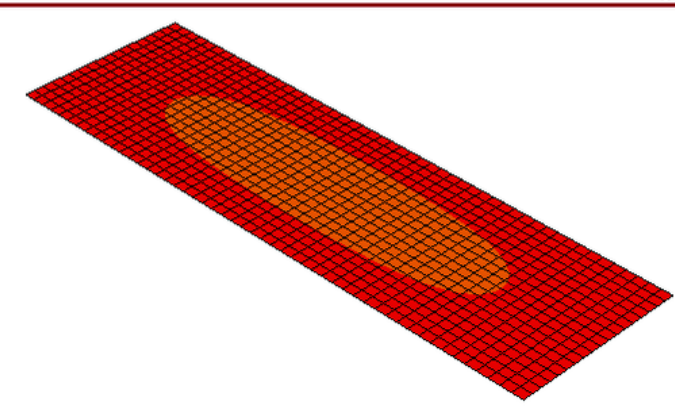

(c)

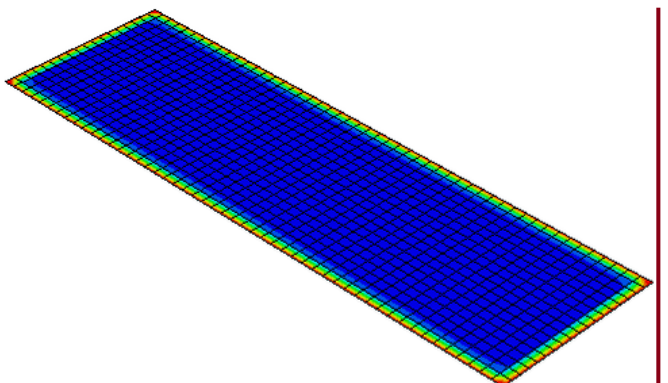

(b)
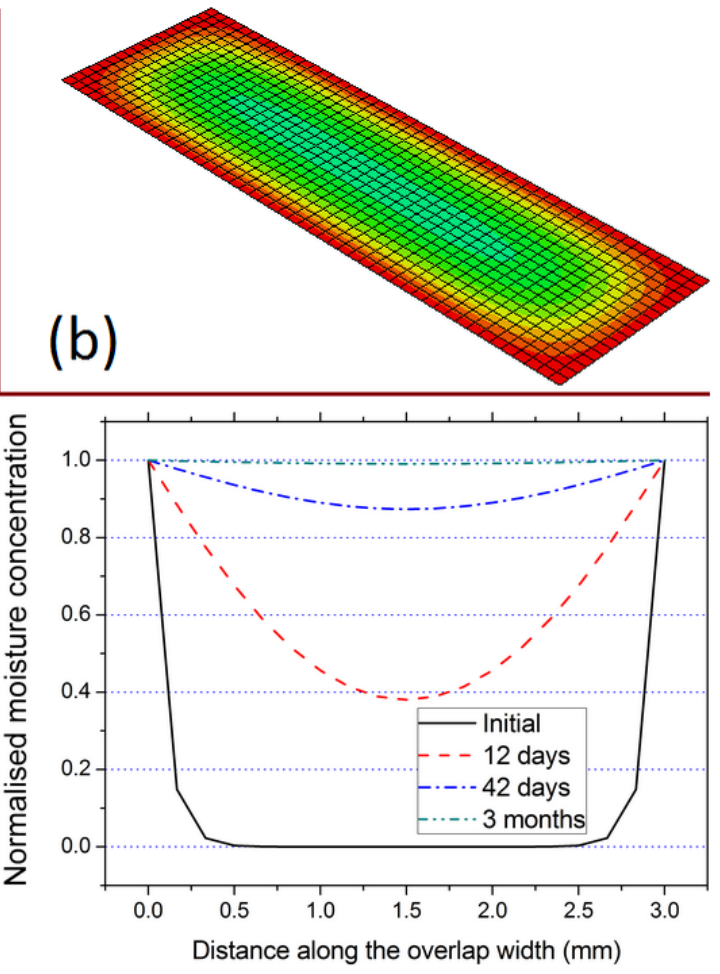

Fig. 6. The moisture concentration states of the adhesive loaded at $12 \%$ of the joint failure strength: moisture contours a) initially, b) after 12 days and c) 42 days and d) distribution along the overlap width.

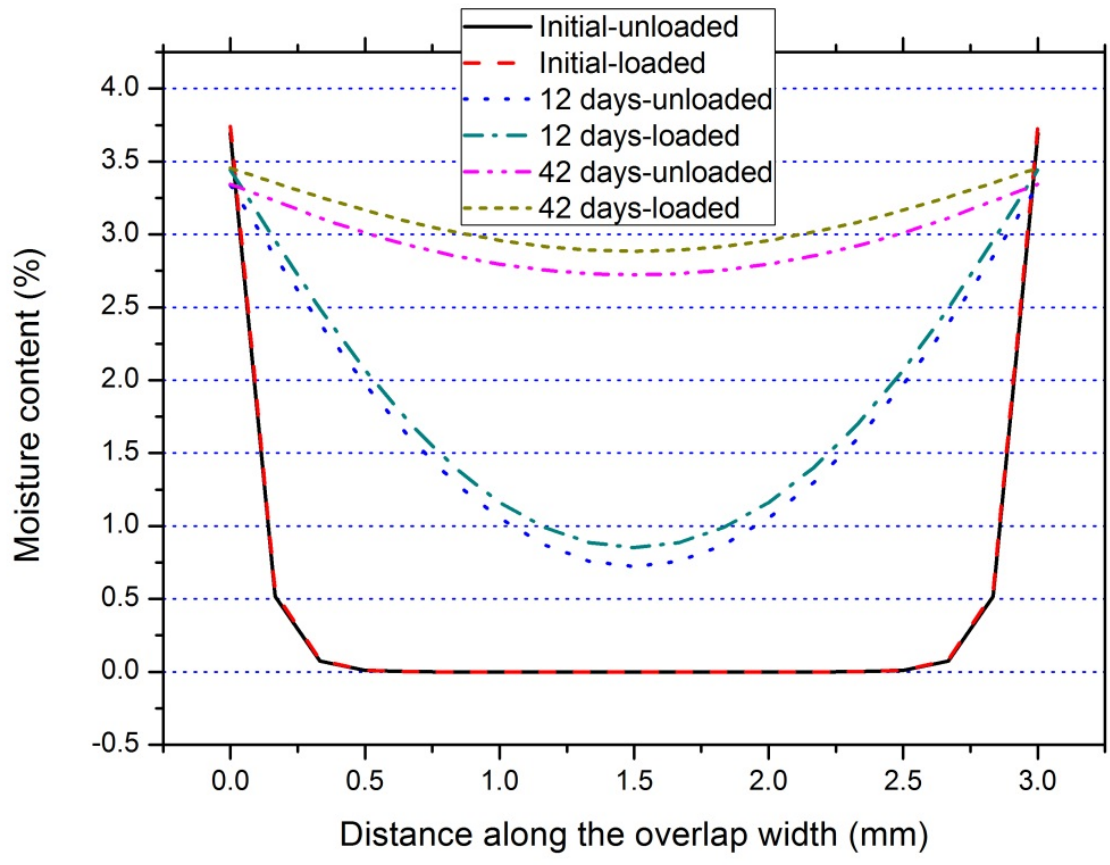

Fig. 7. The moisture content distribution (loaded and unloaded) along the overlap width initially, after 12 days and 42 days. 


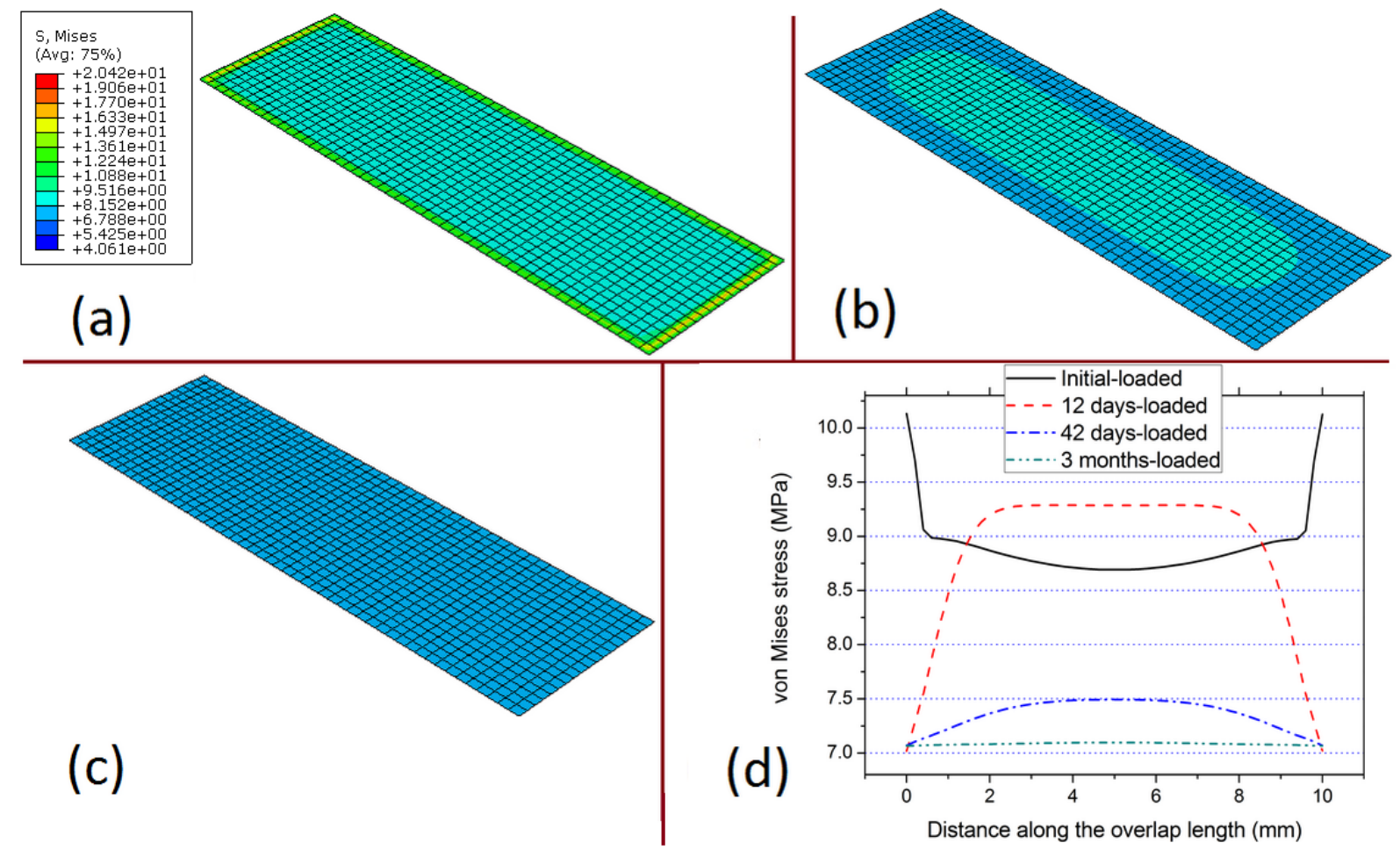

Fig. 8. The von Mises stress states of the adhesive (loaded): a) stress contours initially, b) after 12 days and c) 42 days and d) distribution along the overlap length.

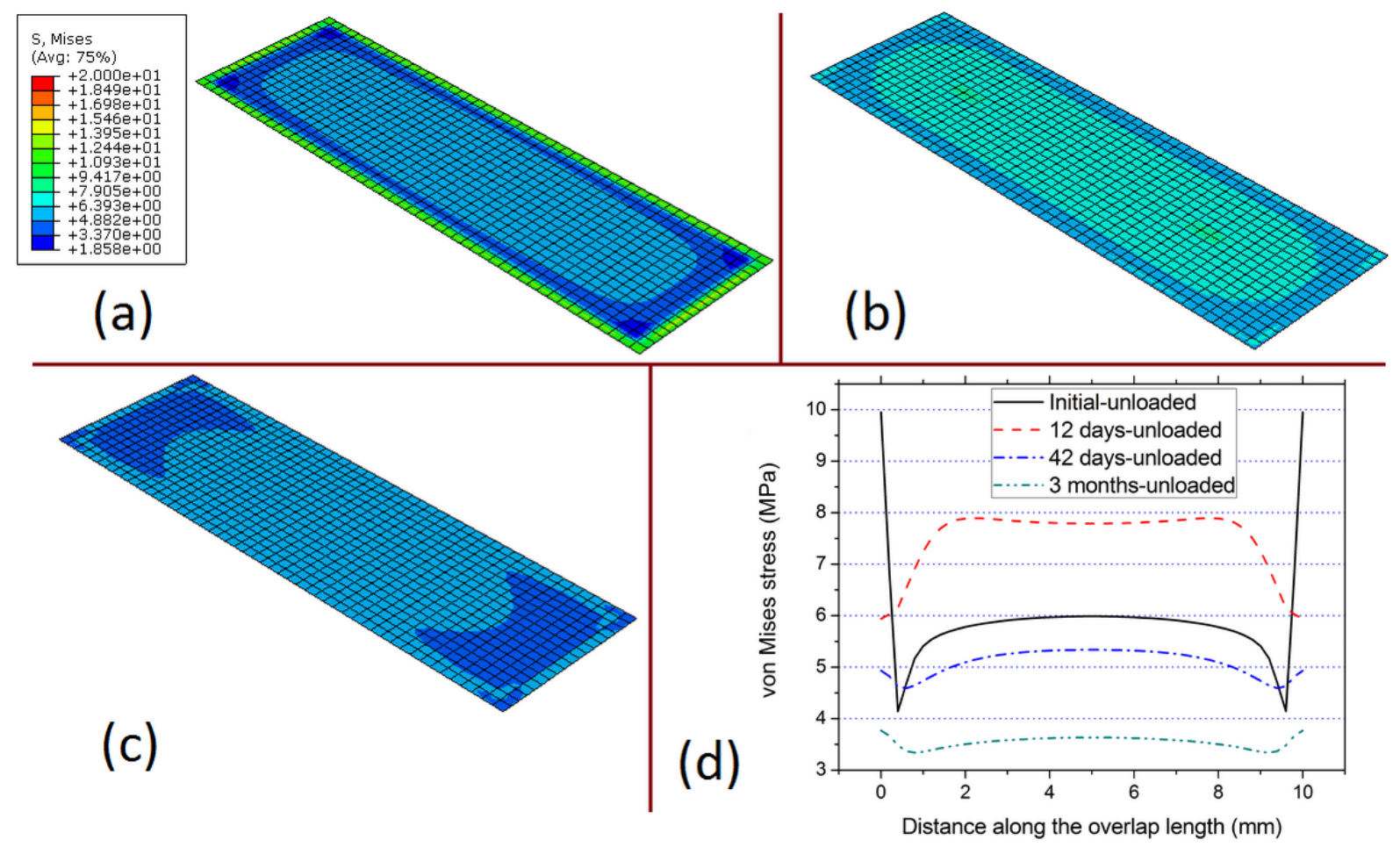

Fig. 9. The von Mises stress states of the adhesive (unloaded): stress contours a) initially, b) after 12 days and c) 42 days and d) distribution along the overlap length. 


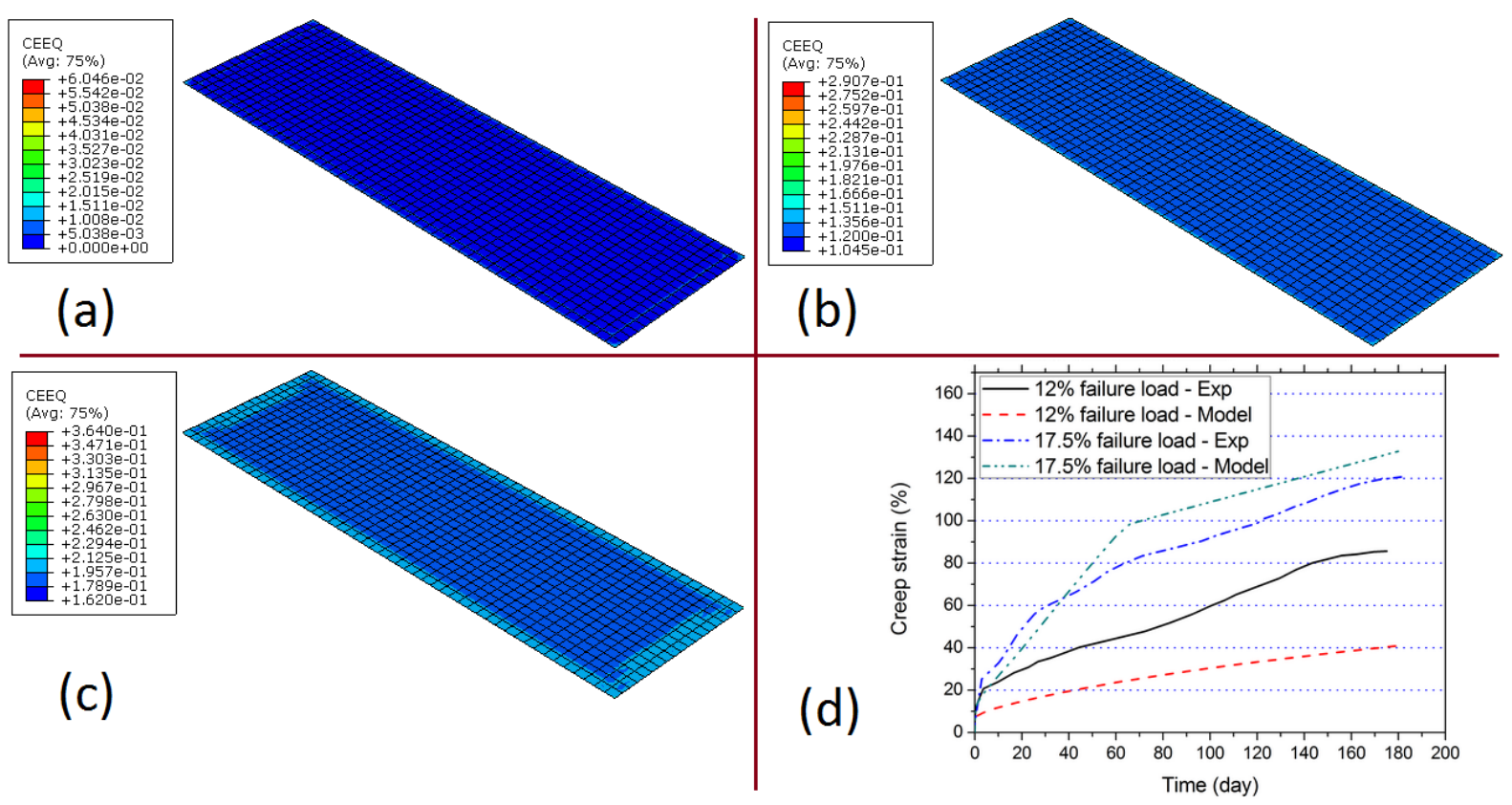

Fig. 10. The equivalent creep strain contours a) initially, b) after 3 months and c) 6 months and d) creep strain-time curves under different loading levels.

\section{Tables}

Table 1 Young's modulus for FM73 at various moisture concentrations.

\begin{tabular}{|c|c|c|}
\hline Environment & Moisture content / \% & Young's Modulus / MPa \\
\hline Dry $/ 50^{\circ} \mathrm{C}$ & 0 & 1650 \\
\hline Immersion $/ 50^{\circ} \mathrm{C}$ & 3.75 & 1400 \\
\hline
\end{tabular}

Table 2 Diffusion parameters for FM73 immersed in $50{ }^{\circ} \mathrm{C}$ deionised water at different loading levels.

\begin{tabular}{|c|c|c|c|}
\hline Stress / MPa & Temperature $/{ }^{\circ} \mathrm{C}$ & Saturation content $/ \%$ & Diffusion coefficient $/ \mathrm{m}^{2} / \mathrm{s}$ \\
\hline 0 & 50 & 2.95 & $5.21 \mathrm{E}-13$ \\
\hline 11.75 & 50 & 3.75 & $7.18 \mathrm{E}-13$ \\
\hline
\end{tabular}

Table 3 Creep parameters for FM73 at $50^{\circ} \mathrm{C}$ (force / N, length / mm, time / s, stress / MPa).

\begin{tabular}{|c|c|c|c|c|}
\hline Phase & Condition & $A$ & $n$ & $m$ \\
\hline First & Dry & $5.774 \mathrm{E}-010$ & 4.75 & -0.4764 \\
\hline First & Saturated & $1.398 \mathrm{E}-009$ & 4.75 & -0.397 \\
\hline Secondary & Partial & $2.956 \mathrm{E}-013$ & 4.75 & 0 \\
\hline
\end{tabular}

